OPEN ACCESS

Edited by:

Andrea Gomez-Zavaglia, Center for Research and Development in Food Cryotechnology (CIDCA, CONICET), Argentina

Reviewed by: Pendru Raghunath, Texila American University, Guyana Barbara Bravo Ferrada, Independant Researcher, Argentina

${ }^{*}$ Correspondence: Roberto Pérez-Torrado rober@iata.csic.es

Specialty section: This article was submitted to Food Microbiology,

a section of the journa

Frontiers in Microbiology

Received: 21 December 2015 Accepted: 03 February 2016

Published: 18 February 2016

Citation:

Navarro-Tapia E, Nana RK, Querol A and Pérez-Torrado R (2016) Ethanol

Cellular Defense Induce Unfolded

Protein Response in Yeast.

Front. Microbiol. 7:189

doi: 10.3389/fmicb.2016.00189

\section{Ethanol Cellular Defense Induce Unfolded Protein Response in Yeast}

\author{
Elisabet Navarro-Tapia, Rebeca K. Nana, Amparo Querol and Roberto Pérez-Torrado * \\ Instituto de Agroquímica y Tecnología de los Alimentos-Consejo Superior de Investigaciones Científicas, Valencia, Spain
}

Ethanol is a valuable industrial product and a common metabolite used by many cell types. However, this molecule produces high levels of cytotoxicity affecting cellular performance at several levels. In the presence of ethanol, cells must adjust some of their components, such as the membrane lipids to maintain homeostasis. In the case of microorganism as Saccharomyces cerevisiae, ethanol is one of the principal products of their metabolism and is the main stress factor during fermentation. Although, many efforts have been made, mechanisms of ethanol tolerance are not fully understood and very little evidence is available to date for specific signaling by ethanol in the cell. This work studied two S. cerevisiae strains, CECT10094, and Temohaya-MI26, isolated from flor wine and agave fermentation (a traditional fermentation from Mexico) respectively, which differ in ethanol tolerance, in order to understand the molecular mechanisms underlying the ethanol stress response and the reasons for different ethanol tolerance. The transcriptome was analyzed after ethanol stress and, among others, an increased activation of genes related with the unfolded protein response (UPR) and its transcription factor, Hac1p, was observed in the tolerant strain CECT10094. We observed that this strain also resist more UPR agents than Temohaya-MI26 and the UPR-ethanol stress correlation was corroborated observing growth of 15 more strains and discarding UPR correlation with other stresses as thermal or oxidative stress. Furthermore, higher activation of UPR pathway in the tolerant strain CECT10094 was observed using a UPR mCherry reporter. Finally, we observed UPR activation in response to ethanol stress in other S. cerevisiae ethanol tolerant strains as the wine strains T73 and EC1118. This work demonstrates that the UPR pathway is activated under ethanol stress occurring in a standard fermentation and links this response to an enhanced ethanol tolerance. Thus, our data suggest that there is a room for ethanol tolerance improvement by enhancing UPR response.

Keywords: yeasts, Saccharomyces, ethanol stress, transcriptomics, UPR

\section{INTRODUCTION}

All living organisms are subjected to changing environmental conditions, such as temperature, humidity, and salinity, which can affect optimal growth and reproduction conditions. Cells have developed diverse strategies to combat the harmful effects of a variety of stress conditions that depend on a complex network of sensors and signal transduction pathways, which lead to adaptation in cell cycle, and also to adjustments in gene expression profiles and cell metabolic activities. Although, Saccharomyces cerevisiae is a traditional ethanol-producing microbe 
widely used for the production of bioethanol, alcoholic beverages, and other industrial products, this yeast is also sensible to ethanol that negatively influences the fermentation kinetics (AnsanayGaleote et al., 2001). Ethanol is a two-carbon alcohol which, due to its small size and alcoholic hydroxyl group, is soluble in both aqueous and lipid environments, which allows it to pass into cells through the plasma membrane increasing fluidity and permeability. Ethanol disturbance of cellular membranes involves a number of consequences that affect many cellular functions (Brooks, 2000; Albano, 2006). Although ethanol forms part of metabolism in many organisms, it alters mitochondrial structure, lowers respiratory rates and ATP levels, and elicits the formation of reactive oxygen species (ROS) and acetaldehyde, which ultimately generate DNA damage, lipid peroxidation and oxidative stress, and reduce cell viability (Brooks, 2000; Hoek and Pastorino, 2002; Lamarche et al., 2003; Albano, 2006; Pandol et al., 2010). Several studies have provided us with some leads to the molecular basis underlying yeast response and resistance to ethanol stress. A correlation between ethanol resistance and trehalose, proline and ergosterol accumulation to enhance the stability of proteins and membranes, and the influence of the degree of fatty acid unsaturation of membrane lipids to antagonize fluidity by ethanol, have been documented (Alexandre et al., 1994a,b; You et al., 2003). Cell wall remodeling, tryptophan biosynthesis, and induction of multiple chaperones and heat shock proteins by the oxidative stress and up-regulation of the genes related with $\mathrm{NADH} / \mathrm{NADPH}$ regeneration to assure a good redox balance have also been reported (Rosa and Sá-Correia, 1991; Alexandre et al., 2001; Hirasawa et al., 2007; Yoshikawa et al., 2009; Li et al., 2010; Stanley et al., 2010a). Ethanol also causes intracellular acidification due to the influx of protons through the damaged cell membrane, which triggers the transport of intracellular $\mathrm{H}^{+}$into vacuoles by $\mathrm{V}$-ATPase to maintain intracellular $\mathrm{pH}$ homeostasis (Rosa and Sá-Correia, 1991; Rosa and Sa-Correia, 1996; Forgac, 1998). Although many efforts have been made, mechanisms of ethanol tolerance activation are not fully understood and very little evidence is available to date for specific signaling by ethanol in the cell. Transcription factor Msn2p and its homologous Msn4p are involved in the ethanol response in $S$. cerevisiae via a stress response element (STRE), although other stresses, like heat, osmotic shock or oxidative stress, activate this general stress response. Transcription factors Yap1p and Hsflp, required for oxidative stress tolerance and heat shock response respectively, are also related to ethanol stress given its pleiotropic effects, which shows that many genes up-regulated by ethanol challenges share the transcription binding motifs of Msn2p/Msn4p, Yap1, Hsf1, and Pdr1p/Pdr3p in their upstream sequence (Ma and Liu, 2010a). Takemura et al. (2004) observed that the nuclear localization of the DEAD box protein Rat8p caused by ethanol stress may contribute to the selective export of mRNA in ethanol-stressed cells.

Over the years, a number of approaches have been used to improve alcohol tolerance to elicit certain cellular phenotypes, such as transposon mutagenesis, gene deletions, and gene transcription reprogramming (Takahashi et al., 2001; Kubota et al., 2004; Alper et al., 2006). More recently, global gene expression studies have provided a better understanding of the molecular basis underlying yeast response and resistance to ethanol stress (Alexandre et al., 2001; Chandler et al., 2004; Li et al., 2010; Stanley et al., 2010a). Although genomewide approaches to reveal ethanol tolerance candidate genes in industrial strains have been adopted (Rossignol et al., 2003; Marks et al., 2008) most studies have focused on laboratory strains with moderate ethanol tolerance and have left aside the physiological diversity of natural or fermentation isolates (Takahashi et al., 2001; Fujita et al., 2006; Teixeira et al., 2009; Stanley et al., 2010a). Furthermore, most of these studies have focused on the response to a short-term ethanol stimulus. However, ethanol stress is a long-term stress with a highly dynamic transcriptional response. Thus, it is essential to study not only early, but also late responses, as there is generally little overlap between the genes transcriptionally induced under stress and those that appear essential for adaptation. In previous studies, we determined significant differences in ethanol tolerance between natural and fermentative $S$. cerevisiae strains, including strains isolated from flor wine and traditional fermentations of Latin America (Arroyo-López et al., 2010).

Considering these data, this work focused on exploiting the physiological characteristics of two fermentative strains, CECT10094 and Temohaya-MI26, isolated from flor wine and agave fermentations, which differ in ethanol tolerance, to understand the molecular mechanisms underlying the ethanol stress response and the reasons for different ethanol tolerance among $S$. cerevisiae strains.

\section{METHODS}

\section{Yeast Growth Media}

The basal growth media selected for the experiments were standard GPY medium (5 g/L yeast extract, $5 \mathrm{~g} / \mathrm{L}$ peptone, $20 \mathrm{~g} / \mathrm{L}$ glucose). Media were modified whenever necessary with geneticine $(200 \mu \mathrm{g} / \mathrm{ml})$, tunycamicine $(1 \mu \mathrm{g} / \mathrm{ml})$, $\mathrm{H}_{2} \mathrm{O}_{2}(3 \mathrm{mM})$, beta-mercaptoethanol $(0-45 \mathrm{mM})$, or ethanol [0 and $10 \%(\mathrm{v} / \mathrm{v})]$.

\section{Strains and Plasmid Construction}

The strains used in this work are listed in Table 1. The $S$. cerevisiae strains used in the genome-wide analysis, CECT10094 and Temohaya-MI26, belong to our collection and were isolated from different traditional fermentation environments (Santa María, 1968; Arroyo-López et al., 2010). In the previous work, Arroyo-López et al. (2010) had a misidentification and described CECT10094 as PE35M. The 15 S. cerevisiae strains used in the correlation study among the different stressors belonged to our own collection. The mCherry UPR reporter with geneticine selective marker was obtained by a marker swap of the commercial plasmid pPM47 (Addgene). KanMX cassette with URA3 extremes was amplified from pFA6a-KanMX6 plasmid (Addgene) and cotransformed with EcoRV digested pRM47 following described procedures 
TABLE 1 | Strains used in this work.

\begin{tabular}{llll}
\hline Strain & Species & Origin & Source \\
\hline CECT10094 & S. cerevisiae & Flor wine (Spain) & Santa María, 1968 \\
Temohaya-MI26 & S. cerevisiae & Agave fermentation (Mexico) & Arroyo-López et al., 2010 \\
CHR9 & S. cerevisiae & Forest soil (Hungary) & Arroyo-López et al., 2010 \\
CECT 1942 & S. cerevisiae & Ale beer (Netherlands) & Belloch et al., 2008 \\
CHR96.2 & S. cerevisiae & Oak tree bark (Spain) & Arroyo-López et al., 2010 \\
RVAC & S. cerevisiae & Wine fermentation (Spain) & Arroyo-López et al., 2010 \\
CPE7 & S. cerevisiae & Sugarcane fermentation (Brazil) & Arroyo-López et al., 2010 \\
CECT 11001 & S. cerevisiae & Lager beer (Belgium) & Oliveira et al., 2014 \\
Lalvin T73C & S. cerevisiae & Wine fermentation (Spain) & Oliveira et al., 2014 \\
GB Flor-C & S. cerevisiae & Wine fermentation (Spain) & Arroyo-López et al., 2010 \\
EC1118C & S. cerevisiae & Champagne (France) & Arroyo-López et al., 2010 \\
D14 & S. cerevisiae & Food supplement & Llopis et al., 2012 \\
60 & S. cerevisiae & Clinical isolate (Spain) & Llopis et al., 2012 \\
W303 & S. cerevisiae & Laboratory strain & Our collection \\
BY4743 & S. cerevisiae & Laboratory strain & Euroscarf
\end{tabular}

${ }^{c}$ Commercial strain.

(Cross, 1997). Plasmids were recovered and confirmed by PCR and enzyme restriction analysis.

\section{Growth Conditions and Experimental Design for Transcriptome Analysis}

A single colony of each strain, CECT10094 and TemohayaMI26, was picked up from fresh GPY-agar plates and separately incubated overnight into $12 \mathrm{~mL}$ sterile tubes with $5 \mathrm{~mL}$ GPY in an orbital shaker at $150 \mathrm{rpm}$ and $28^{\circ} \mathrm{C}$. These cultures in the exponential phase were used to inoculate $100 \mathrm{~mL}$ of GPY to an initial $\mathrm{OD}_{600}$ of 0.1 into sterile $250 \mathrm{~mL}$ Erlenmeyer flasks with cotton wool plugs in the absence or presence of ethanol $10 \%$ $(\mathrm{v} / \mathrm{v})$, a concentration that significantly affects both strains but allow them to grow (Arroyo-López et al., 2010). The samples for RNA extraction were taken after $1 \mathrm{~h}$ (early response) and after exponential phase (late response) under both ethanolstressed and non-stressed conditions. Samples were taken by centrifugation at $3500 \mathrm{rpm}$ for $2 \mathrm{~min}$, washed with water and frozen in liquid $\mathrm{N}_{2}$ (all in less than $5 \mathrm{~min}$.) according to the data obtained from previous observations under the same growth conditions. Cultures, carried out in triplicate, were incubated at $28^{\circ} \mathrm{C}$ and at $150 \mathrm{rpm}$ and the growth profile was monitored using a spectrophotometer (Eppendorf).

\section{RNA Preparation, cDNA Synthesis, and Labeling}

Aliquots for RNA extraction were collected after $1 \mathrm{~h}$ (early response) in both conditions and $10 \mathrm{~h}$ (late response) after inoculation in the absence of ethanol, and 28 and $40 \mathrm{~h}$ for the stressed cultures corresponding to the late response of the CECT10094 and Temohaya-MI26 strains, respectively. Both ethanol-treated and non-treated cells were recovered by centrifugation at $845 \mathrm{~g}$, washed with diethyl pirocarbonatetreated water (Fluka, Taufkirchen, Germany). Pellets were immediately frozen in liquid nitrogen and stored at $-80^{\circ} \mathrm{C}$ until RNA extraction. RNA extraction was carried out according to (Combina et al., 2012). RNA total concentration and purity was quantified using NanoDrop ${ }^{\text {TM }} 1000$ (NanoDrop Technologies, Wilmington, DE). The quality of the total RNA samples also was checked with an agarose gel. Fluorescently labeled complementary DNA (cDNA) was performed with "SuperScript ${ }^{\mathrm{TM}}$ Indirect cDNA Labeling System" (Invitrogen ${ }^{\mathrm{TM}}$, San Diego, CA, USA). Briefly, $20 \mu \mathrm{g}$ of total RNA and $2.5 \mu \mathrm{g}$ oligo $(\mathrm{dT})_{20}$ (Invitrogen) were mixed in $16 \mu \mathrm{l}$ of DEPC water. The solution was heated to $70^{\circ} \mathrm{C}$ for $5 \mathrm{~min}$ and then chilled on ice. Reverse transcription was carried out with SuperScript ${ }^{\mathrm{TM}}$ III Reverse Transcriptase, $334 \mu \mathrm{M}$ aa-dUTP, $6 \mu \mathrm{M}$ oligo (dT), $500 \mu \mathrm{M}$ dATP, dCTP, dGTP, $166 \mu \mathrm{M}$ dTTP, and $10 \mu \mathrm{M}$ DTT in a final volume of $13 \mu \mathrm{l}$. The reaction was incubated at $50^{\circ} \mathrm{C}$ for $12 \mathrm{~h}$ and then with $10 \mu \mathrm{L}$ of $1 \mathrm{M} \mathrm{NaOH}$ and $10 \mu \mathrm{L}$ of $0.5 \mathrm{M}$ EDTA for $15 \mathrm{~min}$ at $70^{\circ} \mathrm{C}$ to degrade the original RNA. $\mathrm{pH}$ were neutralized with $250 \mathrm{mM} \mathrm{HCl}$. Purification of aminoallyl-cDNA (aa-cDNA) was performed with the Mini Elute PCR Purification Kit (Quiagen) according to the manufacturer's instructions. Dye coupling was performed for $2 \mathrm{~h}$ at room temperature and in the darkness with CyDye NHS esters (Amersham Biosciences) and $0.13 \mathrm{M} \mathrm{Na}_{2} \mathrm{CO}_{3}$ to form covalent bonds with the aa-cDNA groups. All the samples were labeled with Cy5 dye, while a reference pool was labeled with $\mathrm{Cy} 3$. The reference pool, which allowed the comparison of the expression differences between any samples (Gasch et al., 2000), was prepared by pooling the RNA extracted in all the samples. The amount of RNA for each sample in the pool was adjusted until approximately equal and a pool was obtained with essentially equimolar amounts of each sample. Purified labeled cDNA was then tested to verify the dye incorporation efficiency and quality using NanoDrop ${ }^{\mathrm{TM}}$ 1000. Only those samples above 100 pmol were used in the assay. No dye-swapping was carried out as the proportion of genes that had a bias in $\mathrm{Cy} 3$ or $\mathrm{Cy} 5$ incorporation was around $0.1 \%$, and this problem is more common when cDNA 
labeling is performed by the direct method (Causton et al., 2003).

\section{Microarrays Hybridization, Scanning, and Data Analysis}

The pre-hybridization step was performed in 3X SSC solution (Sigma), $0.1 \%$ SDS (Sigma) and $0.1 \mathrm{mg} / \mathrm{ml}$ BSA for $60 \mathrm{~min}$ at $50^{\circ} \mathrm{C}$ to minimize background noise and to remove friable DNA probes. Competitive hybridization was carried out manually using an equal quantity of the two labeled samples (100-200 pmol) concentrated in a Concentrator Plus (Eppendorf ${ }^{\mathrm{TM}}$, Hamburg, Germany). The mixture was resuspended in hybridization solution (5x SSC, $0.1 \%$ SDS, $50 \%$ formamide and $0.1 \mathrm{mg} / \mathrm{mL}$ salmon sperm DNA), dropped onto the Yeast $6.4 \mathrm{~K}$ Array (Microarray Centre, UHN, Toronto, Ontario, Canada) and covered with coverslips HybriSlip (Grace Bio-Labs, Sigma). Hybridization was conducted for $16 \mathrm{~h}$ in AHC chambers (Arraylt Corporation, CA, USA) and immersed in a bath at $42^{\circ} \mathrm{C}$, and the labeled microarrays were washed manually with different solutions containing different SSC and SDS concentrations (Sol.1: $2 \mathrm{x}$ SSC, $0.1 \%$ SDS for $5 \mathrm{~min}$ at $42^{\circ} \mathrm{C}$; Sol 2: $0.1 \mathrm{x}$ SSC, $0.1 \%$ SDS for $5 \mathrm{~min}$ at room temperature; Sol 3: $0.1 \mathrm{x}$ SSC for $5 \mathrm{~min}$ at room temperature; Sol 4: $0.01 \%$ SSC for $10 \mathrm{~s}$ ), dried by centrifugation at $135 \mathrm{~g}$ for $10 \mathrm{~min}$ and stored in the dark. Each microarray came from a biological replicate and 24 slides were obtained.

The signal intensities of Cy 3 and Cy5 were acquired with an Axon GenePix 4100 scanner (Axon Instruments, Foster city, CA, USA). Flawed or poor quality spots were manually removed from the data set and a global background subtraction was done by GenePix Pro 6.0 (Molecular Devices Corp., Union City; CA, USA). The expression ratio values (Cy5/Cy3) were transformed into logarithm base 2 to treat up- and down-regulated genes equivalently. Raw microarray data were analyzed by Acuity 4.0 (Molecular Devices Corp.), the fluorescence intensity corresponding to the two dyes was ratio-based normalized and quality control conditions were applied to remove unreliable data from the analysis. Replicates were combined and their medians were calculated, and only the spots with at least two replicates were considered. To identify the genes whose variation was due to the effect of ethanol, an indirect comparison between the slides corresponding to stressed and unstressed samples was made. The genes differentially expressed at the $95 \%$ confidence level were identified as being $\log _{2}$ (ratio) values with more than 1.96 standard deviations from the mean (Causton et al., 2003). A 5\% False Discovery Rate was applied to correct for any false positives appearing. Microarray data was validated by $\mathrm{qPCR}$ (Supplementary Figure 1).

The enrichment of the functional categories among the up-regulated genes was analyzed using the web tool GOTermFinder (http://go.princeton.edu/cgi-bin/GOTermFinder) and by employing Bonferroni's correction and a $p$-value threshold $<0.05$. For the purpose of summarizing and removing redundant GO terms, the web server was used REViGO (Supek et al., 2011), which uses a simple clustering algorithm that relies on semantic similarity measures.

\section{Growth Analysis under Unfolded Protein Stress in the CECT10094 and Temohaya-MI26 Strains}

Inocula were prepared by introducing a single colony from the pure cultures of each strain into $5 \mathrm{~mL}$ of GPY. After overnight incubation at $28^{\circ} \mathrm{C}, 1 \mathrm{~mL}$ of each tube was centrifuged at $845 \mathrm{~g}$ for $5 \mathrm{~min}$, and pellets were washed with sterile saline solution $(0.9 \% \mathrm{NaCl})$, centrifuged and diluted to an optical density $\left(\mathrm{OD}_{600}\right)$ of $0.15-0.2$ in $250 \mu \mathrm{l}$ of GPY medium modified with different concentrations $(0-45 \mathrm{mM})$ of $\beta$-mercaptoethanol (Sigma-Aldrich). Growth was monitored in 96-well plates at $600 \mathrm{~nm}$ for $72 \mathrm{~h}$ in a SPECTROstar Omega instrument (BMG Labtech, Offenburg, Germany); measurements were taken every $30 \mathrm{~min}$ after pre-shaking for $40 \mathrm{~s}$. All the experiments were carried out in triplicate under aerobic conditions and uninoculated wells for each experimental series were also included to subtract the noise signal. In this way, 114 growth curves were obtained and analyzed. In order to obtain a quantitative methodology that allows an objective and reliable comparison among yeasts, a modified Gompertz equation for decay was used to objectively estimate the NIC and the MIC for the experiments with BME (Lambert and Pearson, 2000). These parameters are related to the susceptibility and resistance of yeast to this compound, respectively. To check for significant differences for the NIC and MIC parameters, an unpaired $t$-test was performed using GraphPad Prism, version 5.0, for Windows (GraphPad Software, San Diego California USA).

\section{Correlation Analysis between Ethanol Tolerance and other Stresses}

We analyzed the growth of $15 \mathrm{~S}$. cerevisiae strains of different origins (Table 1) in the GPY medium modified with different stressors: $1 \mu \mathrm{g} / \mathrm{mL}$ tunycamicine (Sigma-Aldrich), 10\% (v/v) ethanol (Scharlau Chemie S.A., Spain), $30 \mathrm{mM} \mathrm{BME} \mathrm{(Sigma-}$ Aldrich), $3 \mathrm{mM}$ oxygen peroxide (Merck Millipore), and GPY medium at $\mathrm{pH}$ 10. Growth was monitored with a SPECTROstar Omega instrument (BMG Labtech, Offenburg, Germany). In order to correlate ethanol tolerance with other kinds of tolerance (unfolded protein tolerance, oxidative stress tolerance, etc.), we determined the area under the $\mathrm{OD}_{600}$-time curve as a measure of overall yeast growth in all the strains and under all the conditions. The areas under the $\mathrm{OD}_{600}$-time curves were calculated by integration using the OriginPro 7.5 software (OriginLab Corporation, Northampton, USA). The relative amount of growth for each stressor was obtained by following this formula:

$$
f a=\left(\text { area }_{\text {test }}\right) /\left(\text { area }_{\text {cont }}\right)
$$

where $f a$ is denoted as the fractional area; areatest is the test area

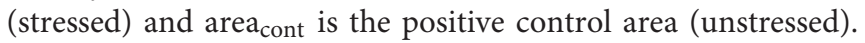
We performed a correlation analysis from the plots of the $f a$ (ethanol) vs. fa (each stressor) using GraphPad Prism, version 5.0, for Windows (GraphPad Software, San Diego California USA). 


\section{UPR Reporter Assays}

To measure UPR activity under ethanol stress we used a modified plasmid reporter called UPR-mCherry that encodes red fluorescent protein mCherry (Merksamer et al., 2008), driven by a minimal CYC1 promoter and four tandem unfolded protein response elements. Cells containing the plasmid were grown overnight in GPY with geneticine medium at $28^{\circ} \mathrm{C}$ and were allowed to reach the early exponential phase (an approximate OD600 value of 0.4 ) for the analysis. Then the culture was divided into sterile centrifuge tubes, pelleted and incubated with GPY media with geneticine, with or without $10 \%(\mathrm{v} / \mathrm{v})$ ethanol. Cells were grown at $28^{\circ} \mathrm{C}$, sampled every $2 \mathrm{~h}$, pelleted and frozen in liquid nitrogen until use. GFP fluorescence was measured by flow cytometry in a LSR Fortessa flow cytometer (BD Biosciences) and analyzed with the FACS DIVA software to compile.fcs files. Files were analyzed using FloJo (Tree Star Ashland, OR). Median fluorescence intensities (MFI) were calculated for each channel and were normalized with time cero sample data. To quantify UPR induction, fractional area (fa) of $10 \%$ ethanol condition was normalized against control (see above). Biological triplicates were performed in all cases.

\section{Availability of Supporting Data}

The original data from this study are available from GEO (http:// www.ncbi.nlm.nih.gov/geo/) with accession number GSE44863.

\section{RESULTS}

\section{Growth of Ethanol Tolerant and Sensitive S. cerevisiae Strains}

In order to elucidate transcriptional differences under ethanol stress in yeast isolates, we focused on two S. cerevisiae strains, CECT10094, and Temohaya-MI26. These strains were selected because they displayed very different ethanol susceptibility and resistance in minimal media (29). We evaluated the growth of these strains in GPY medium (Figure 1A) and GPY with $10 \%(\mathrm{v} / \mathrm{v})$ ethanol (Figure 1B). Our results showed that ethanol resulted in a lower growth rate and an increased lag phase in both strains. Furthermore, we observed a greater growth inhibition in Temohaya-MI26 than CECT10094. The ethanol tolerant strain CECT10094 reached the exponential phase $18 \mathrm{~h}$ before Temohaya-MI26, and obtained a maximum specific growth rate $\left(\mathrm{h}^{-1}\right)$ of 0.50 , unlike Temohaya-MI26 whose maximum specific growth rate was 0.34 . The lag period increased from $1 \mathrm{~h}$ at $0 \%$ ethanol to around 14 and $32 \mathrm{~h}$ at $10 \%$ ethanol for the CECT10094 and Temohaya-MI26 strains, respectively. In contrast, it is interesting to note that in the absence of ethanol, the Temohaya-MI26 strain reaches a $24 \%$ higher $(p<0.05)$ final population than the ethanol-tolerant strain. Therefore in view of the results, the selection of these strains was suitable to study transcriptional differences under ethanol stress in early and late growth stages.

\section{Global Gene Expression Analysis in Response to Ethanol Stress}

To measure global changes in the gene expression levels of tolerant and sensible strains in early and late ethanol stress
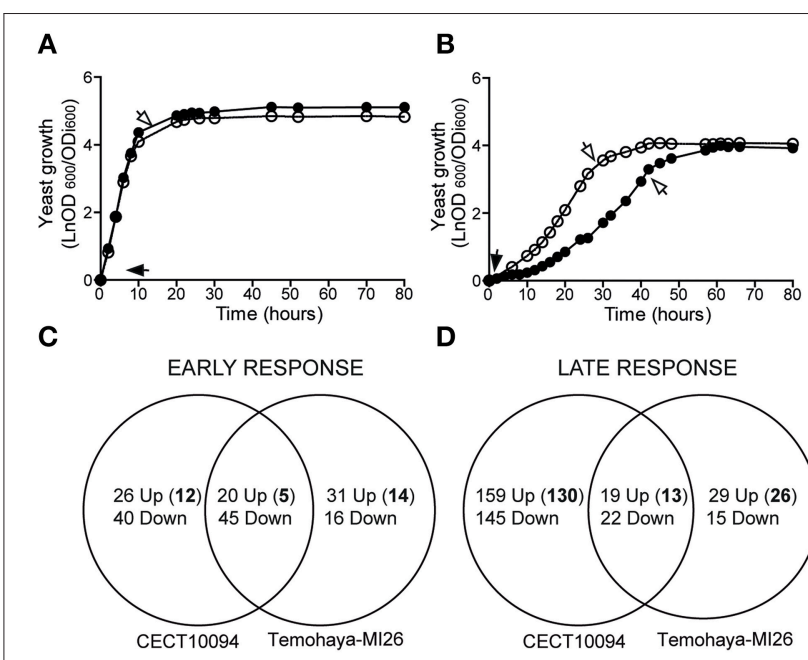

D

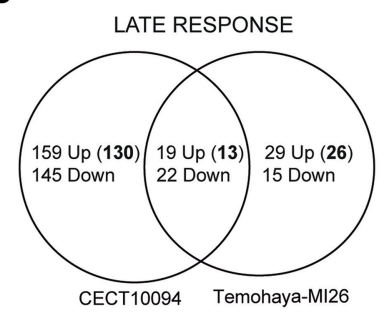

FIGURE 1 | Growth of S. cerevisiae CECT10094 (०) and Temohaya-MI26 (•) on liquid GPY medium containing $0 \%(A)$ or $10 \%(B)$ ethanol. Overnight yeast cells were transferred to $250 \mathrm{~mL}$ flasks containing $100 \mathrm{~mL}$ of GPY, modified or not with $10 \%$ ethanol (v/v), and initial cell density was adjusted to $\mathrm{OD}_{600}=0.1$. Arrows indicate the sampling time for the transcriptome study in the early (black arrows) and late (white arrows) response phase. Cultures were performed in triplicate and incubated at $28^{\circ} \mathrm{C}$ at $150 \mathrm{rpm}$. (C,D) Venn diagram showing the number of genes up- and down-regulated in the CECT10094 and Temohaya-MI26 strains in the early (C) and late response (D) after 10\% (V/v) of ethanol addition. Bold numbers indicate the up-regulated genes not seen in previous works. For more details refer to Supplementary Table 2.

stages, the CECT10094 and Temohaya-MI26 strains were grown at 0 and $10 \%(\mathrm{v} / \mathrm{v})$ ethanol in GPY medium. Gene expression variation in $10 \%(\mathrm{v} / \mathrm{v})$ ethanol respect to $0 \%$ of both strains was compared in the early $(1 \mathrm{~h}$ ) or late (after exponential phase) response to ethanol stress (see arrows in Figure 1). After filtering and normalization, a complete dataset of genes, considered to be significantly down- or up-regulated, was obtained (Supplementary Table 1). In the early response, 178 genes were differentially expressed in both strains (Figure 1C). A total of 46 genes were up-regulated in the tolerant strain, of which 26 were exclusively up-regulated in this strain and some were related with the cellular response to oxidative stress (YDL124W, GRE3 and NCE103), transport and utilization of proline (PUT4 and PUT1), synthesis of mono-unsaturated fatty acids (OLE1), molecular chaperones like HSP104 and HSP32, protein folding (MPD1) or energy reserve metabolic processes (RGI1), and have already been described as key ethanol-tolerance genes. Both strains shared 20 up-regulated genes, the majority of which were related with Heat Shock Proteins (HSP42, HSP12, HSP26, HSP31, and HSP36), acetyl-CoA biosynthesis, glycolysis, and nitrogen utilization. In the Temohaya-MI26 strain, specifically up-regulated genes (Shobayashi et al., 2007) were related with the stress response (DDR2, ALD3, and GRE1), sporulation, cell wall organization and unknown function genes. As expected, after $1 \mathrm{~h}$ of ethanol stress, a large number of down-regulated genes encoded components of the protein synthesis machinery. This group represented 64.3 and $70.5 \%$ of all the down-regulated genes 
in CECT10094 and Temohaya-MI26, respectively. All the upregulated genes after $1 \mathrm{~h}$ of ethanol stress for both strains are compiled in Supplementary Table 2.

In the late response, 389 genes were differentially expressed in both strains (Figure 1D). A total of 178 genes were up-regulated in the CECT10094 strain, of which 159 were exclusively upregulated in this strain. Some were related to protein folding (ERO1, LHS1, SBA1, and SSE2), ATP synthesis (ATP1-4, ATP7, and ATP20), ergosterol biosynthesis (ERG5 and ERG20), heat shock protein (HSP104, HSP31, and HSP82), long-chain fatty acid transport (FAT1), phosphatidylcholine biosynthesis (CHO2), vacuolar acidification (VMA8 and VMA10), Ty element transposition (YMR051C, YJR028W, YAR009C, YJR026W, YML045W, YML040W, YMR046C, YAR010C, and YCLO20W), removal of superoxide radicals (CUP1-1), and protein degradation (CDC34, PRE1, PRE4, PRE6-7, PUP2, $R P N 12, R P N 5, R P N 8, R P T 4-5, S C L 1$, and UBC1). The two strains shared 19 up-regulated genes, which were mainly related with the response to stress (HSC82, YHB1, and MSN4), cell wall organization and biogenesis (YGP1 and ECM4) and amino acid transport (BAP2 and TAT1). In the Temohaya-MI26 strain, there were 29 specifically up-regulated genes, some of which were related with the alcohol metabolic process (ADH6), heat shock protein (HSP12) and sporulation (UBX6 and DIT1). All the up-regulated genes in the late response for both strains are compiled in Supplementary Table 2. It is noteworthy that in the genes expressed only in tolerant strain CECT10094 after $1 \mathrm{~h}$ under ethanol stress, the GO-Term analysis identified GO categories that were related to the response to oxidative stress, proline metabolic process and thiaminecontaining compound biosynthesis, unlike the less tolerant strain, which did not present GO terms related with known ethanol tolerance mechanisms (Table 2). In the late response, CECT10094 showed GO terms related with the protein folding, proteosomal ubiquitin-independent catabolic process, RNAmediated transposition, ATP synthesis coupled proton transport, and proton transport. At this time point, Temohaya-MI26 did not show GO terms relate with ethanol tolerance mechanisms either.

It should be noted that, although more genes were upregulated in the late response (207 genes), only $17.96 \%$ of them were associated with the ethanol stress response in previous studies with microarrays, while the up-regulation of $59.74 \%$ of our genes in the early response (77 genes) has been observed in previous studies (Supplementary Table 2), probably because most microarrays studies done to date have focused on early stages of ethanol stress response.

\section{Unfolded Protein Response and Ethanol Stress}

Taking into account the presence of up-regulated genes associated with the accumulation of unfolded proteins and GO categories related with protein refolding in the most tolerant strain under ethanol stress, we explore the possibility that the unfolded protein response, mediated by transcription factor Haclp, was implicated in ethanol tolerance. Based on this premise, we carried out a promoter analysis of those genes overexpressed specifically in each strain under ethanol stress to determinate the percentage of the up-regulated genes directly related with transcription factor Haclp during ethanol stress. A search in the YEASTRACT database revealed that $19.23 \%$ of the genes exclusively overexpressed in the early phase of ethanol stress and $5.73 \%$ of the genes exclusively overexpressed in the late phase of ethanol stress presented regulatory associations with Haclp in the most ethanol-tolerant strain CECT10094 while Haclp regulated genes were not found in the TemohayaMI26 strain in either growth stage (Table 3). This evidence suggests that increased tolerance to ethanol can be related to an enhanced unfolded protein response (UPR). Other stress-related transcription factors, such as Msn2p/Msn4p for general stress, Hsflp for heat stress, and Yaplp for oxidative stress, were also found (Table 3).

In order to correlate the Haclp-mediated UPR response with ethanol tolerance, we studied the growth of the TemohayaMI26 and CECT10094 strains at different concentrations of endoplasmic reticulum (ER) stressor $\beta$-mercaptoethanol (BME), which prevents disulfide-bond formation. The results showed that Temohaya-MI26 plate growth was significantly affected with the presence of 5 but especially with $15 \mathrm{mM}$ of BME in GPY medium (Figure 2A). In contrast, CECT10094 growth was not significantly affected by BME. With the purpose of clarify this physiological difference among both strains we estimated the non-inhibitory concentration (NIC) and the minimum inhibitory concentration (MIC) by studying yeasts growth in flasks with a range of BME concentrations. Figure $2 \mathbf{B}$ shows the curve fitting for both strains with an $R^{2}$ ranging from 0.96 to 0.99 . The CECT10094 and Temohaya-MI26 strains gave significant differences in the NIC values of $6.54 \pm 1.48 \mathrm{mM}$ and $2.80 \pm$ $0.90 \mathrm{mM}$, and in the MIC values of $36.74 \pm 2.96 \mathrm{mM}$ and $7.29 \pm$ $2.04 \mathrm{mM}$, with a $p$-value of 0.01 and 0.0001 for the NIC and MIC values, respectively. Hence the Temohaya-MI26 strain gave lower NIC and MIC values than the CECT10094 strain, which is indicative of higher susceptibility to the protein denaturant BME. This experiment suggests that strains with an enhanced UPR also have increased ethanol tolerance.

To further ascertain the correlation between UPR and ethanol tolerance, we analyzed the growth of $15 \mathrm{~S}$. cerevisiae strains in GPY medium modified with different stressors, such as tunycamicine, ethanol, BME, oxygen peroxide, and GPY medium at $\mathrm{pH}$ 10. Then we studied the correlation between ethanol and ER stress and compared it to other stresses, such as oxidative, osmotic, and high $\mathrm{pH}$ stress. The correlation analysis (Table 4) showed that tolerance to ethanol and protein folding stress (BME and tunycamicine) correlated significantly, whereas other stresses showed no correlation.

After confirm the physiological relation among ethanol stress and unfolded protein response we wanted to determine if ethanol stress induced UPR activation. To test this hypothesis we used a previously described mCherry UPR reporter containing four Haclp binding sites. After marker swap, we introduced the plasmid with the reporter in the Temohaya-MI26 and CECT10094 strains. Finally, modified strains were inoculated in complete media with or without $10 \%$ ethanol and samples 
TABLE 2 | Enrichment of the functional categories of the up-regulated genes during ethanol stress in the CECT10094 and Temohaya-MI26 strains ${ }^{\text {a }}$.

\begin{tabular}{|c|c|c|c|c|}
\hline GO ID & Biological process & $p$-value & Up-regulated genes & Total genes \\
\hline \multicolumn{5}{|c|}{ EARLY PHASE } \\
\hline \multicolumn{5}{|c|}{ Up-regulated genes in the CECT10094 strain under ethanol stress } \\
\hline GO:0019321 & Pentose metabolic process & 0.0170 & 3 & 14 \\
\hline GO:0055114 & Oxidation-reduction process & 0.0001 & 14 & 450 \\
\hline GO:0006560 & Proline metabolic process & 0.0393 & 2 & 9 \\
\hline \multicolumn{5}{|c|}{ Up-regulated genes in the Temohaya-MI26 strain under ethanol stress } \\
\hline GO:0055114 & Oxidation-reduction process & 0.0001 & 15 & 450 \\
\hline GO:0016052 & Carbohydrate catabolic process & 0.0048 & 7 & 120 \\
\hline GO:0046365 & Monosaccharide catabolic process & 0.0214 & 5 & 66 \\
\hline GO:0010499 & Proteasomal ubiquitin-independent protein catabolic process & 0.0005 & 6 & 14 \\
\hline GO:0032196 & Transposition & 0.0053 & 13 & 114 \\
\hline GO:0032197 & Transposition, RNA-mediated & 0.0190 & 12 & 110 \\
\hline GO:0055114 & Oxidation-reduction process & 0.0105 & 28 & 450 \\
\hline GO:0090342 & Regulation of cell aging & 0.0459 & 3 & 4 \\
\hline GO:0006122 & Mitochondrial electron transport, ubiquinol to cytochrome c & 0.0031 & 5 & 11 \\
\hline GO:0015986 & ATP synthesis-coupled proton transport & 0.0062 & 7 & 30 \\
\hline GO:0042026 & Protein refolding & 0.0375 & 5 & 17 \\
\hline GO:0022900 & Electron transport chain & 0.0036 & 10 & 64 \\
\hline GO:0015992 & Proton transport & 0.0293 & 7 & 42 \\
\hline \multicolumn{5}{|c|}{ Up-regulated genes in the Temohaya-MI26 strain under ethanol stress } \\
\hline
\end{tabular}

${ }^{a}$ GO categories were analyzed using the GO-TermFinder web tool (http://go.princeton.edu/cgi-bin/GOTermFinder) and employing Bonferroni's correction and a p-value threshold of 0.05 , redundant GO terms were removed using the REViGO web server (http://revigo.irb.hr).

TABLE 3 | A promoter region analysis showing $\%$ of the up-regulated genes containing binding sites exclusively in the CECT10094 and Temohaya-MI26 strains after addition of ethanol.

\begin{tabular}{lccccc}
\hline Transcription Factor & \multicolumn{2}{c}{ CECT10094 } & & \multicolumn{2}{c}{ TEMOHAYA-MI26 } \\
\cline { 2 - 3 } & Early (\%) & Late (\%) & & Early (\%) & Late (\%) \\
\hline Msn2p & 65.4 & 32.5 & & 90.3 & 35.7 \\
Msn4p & 65.4 & 28.0 & & 87.1 & 42.9 \\
Hsf1p & 61.5 & 17.2 & & 48 & 17.9 \\
Hac1p & 19.2 & 5.7 & & 0.0 & 0.0 \\
Yap1p & 53.9 & 29.3 & & 45.2 & 39.3 \\
\hline
\end{tabular}

Promoter regions were analyzed by YEASTRACT database in order to find transcription factor-binding sites.

were taken to observe UPR activation by flow cytometry. The results (Figure 3A) show that any strain increased mCherry levels in complete media without ethanol during the first $6 \mathrm{~h}$. Also, almost no activation was observed in Temohaya-MI26 strain with $10 \%$ ethanol in the media. However, the ethanol tolerant strain CECT10094 increased mCherry levels when 10\% ethanol was present in the media, reaching a maximal value of 2.2 -fold after $6 \mathrm{~h}$. This result confirmed that ethanol stress activates UPR, especially in ethanol tolerant strains.

The different levels of UPR activation after ethanol stress in Temohaya-MI26 and CECT10094 prompted us to investigate whether other ethanol tolerant strains have an increased UPR after ethanol stress. To evaluate this hypothesis we selected two different industrial $S$. cerevisiae wine strains previously characterized as ethanol tolerant strains, T73 and EC1118. First we evaluated ethanol tolerance by following cell growth after $10 \%$ ethanol stress (Figure 3B). The results show that wine strains show high levels of ethanol tolerance as CECT10094, comparing to Temohaya-MI26. Next we wanted to evaluate UPR activation in response to ethanol in the same strains and for that we performed the same experimental set up as described previously for CECT10094 and Temohaya-MI26 (Figure 3C). The UPR mCherry reporter activation for all the strains was quantified 


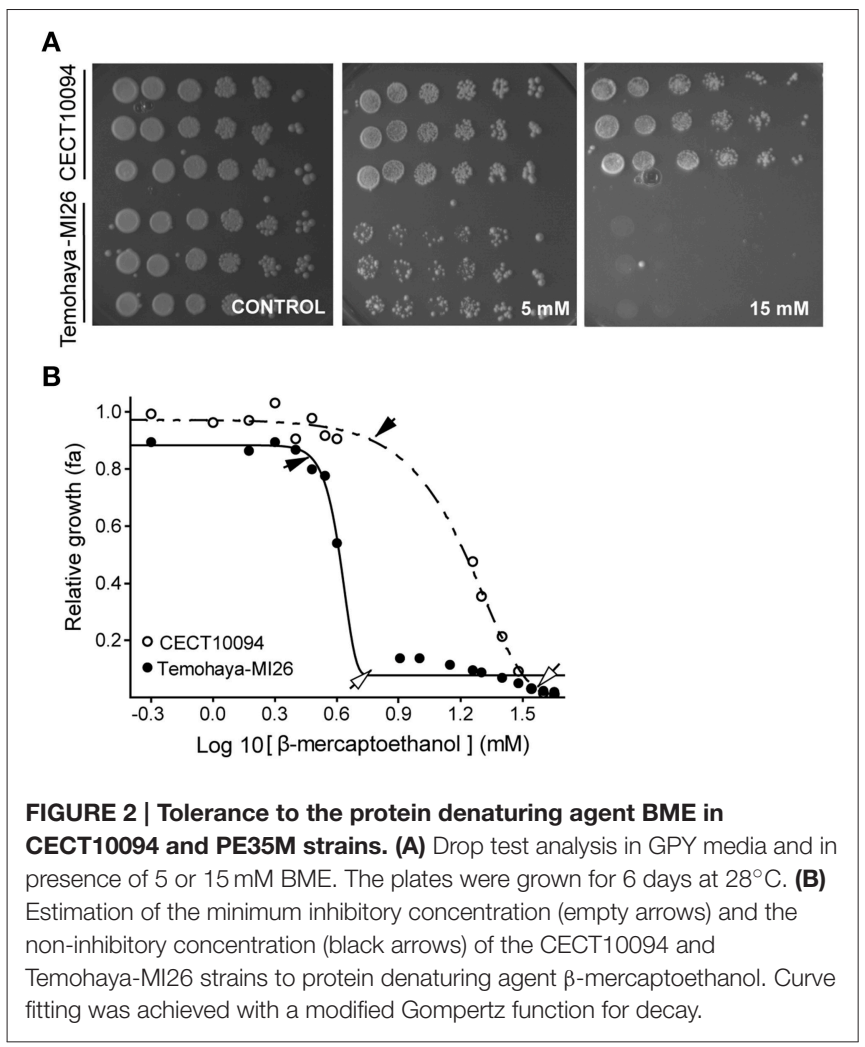

TABLE 4 | Correlation between yeast population behavior after treatment with different agents.

\begin{tabular}{lccccc}
\hline & $\begin{array}{c}\mathbf{1 0 \%} \\
\text { Ethanol }\end{array}$ & $\begin{array}{c}\mathbf{B M E} \\
(\mathbf{3 0} \mathbf{m M})\end{array}$ & $\begin{array}{c}\mathbf{H}_{\mathbf{2}} \mathbf{O}_{\mathbf{2}} \\
\mathbf{( 3 \mathbf { ~ m M } )}\end{array}$ & $\mathbf{p H ~ 1 0}$ & TM $(\mathbf{1} \mu \mathbf{g} / \mathbf{m L})$ \\
\hline $10 \%$ Ehanol & - & $\mathbf{0 . 0 2}$ & 0.29 & 0.05 & $\mathbf{0 . 0 1}$ \\
$\mathrm{BME}(30 \mathrm{mM})$ & & - & 0.08 & 0.24 & $\mathbf{0 . 0 1}$ \\
$\mathrm{H}_{2} \mathrm{O}_{2}(3 \mathrm{mM})$ & & & - & 0.05 & 0.12 \\
$\mathrm{pH} \mathrm{10}$ & & & & - & 0.08 \\
$\mathrm{TM}(1 \mu \mathrm{g} / \mathrm{mL})$ & & & & & -
\end{tabular}

Bold numbers indicate correlation between stressors $(p<0.05)$.

and represented in Figure 3C. The results showed that strains with high ethanol tolerance also present UPR activation whereas Temohaya-MI26, showing low ethanol tolerance, showed almost no UPR activation. These results also point to a functional role of the UPR in the ethanol tolerance in yeast cells and suggest substantial degree of phenotypical diversity among the different $S$. cerevisiae strain types regarding UPR after cytotoxic ethanol effects.

\section{DISCUSSION}

\section{Early and Late Response to Ethanol Stress}

In the present work, we analyzed yeast responses against ethanol stress in two strains which differed in terms of ethanol tolerance in two growth stages. Our results revealed that optimal tolerance of yeasts to ethanol implied a series of dynamic events prolonged in the time rather than a short transient response. Our results
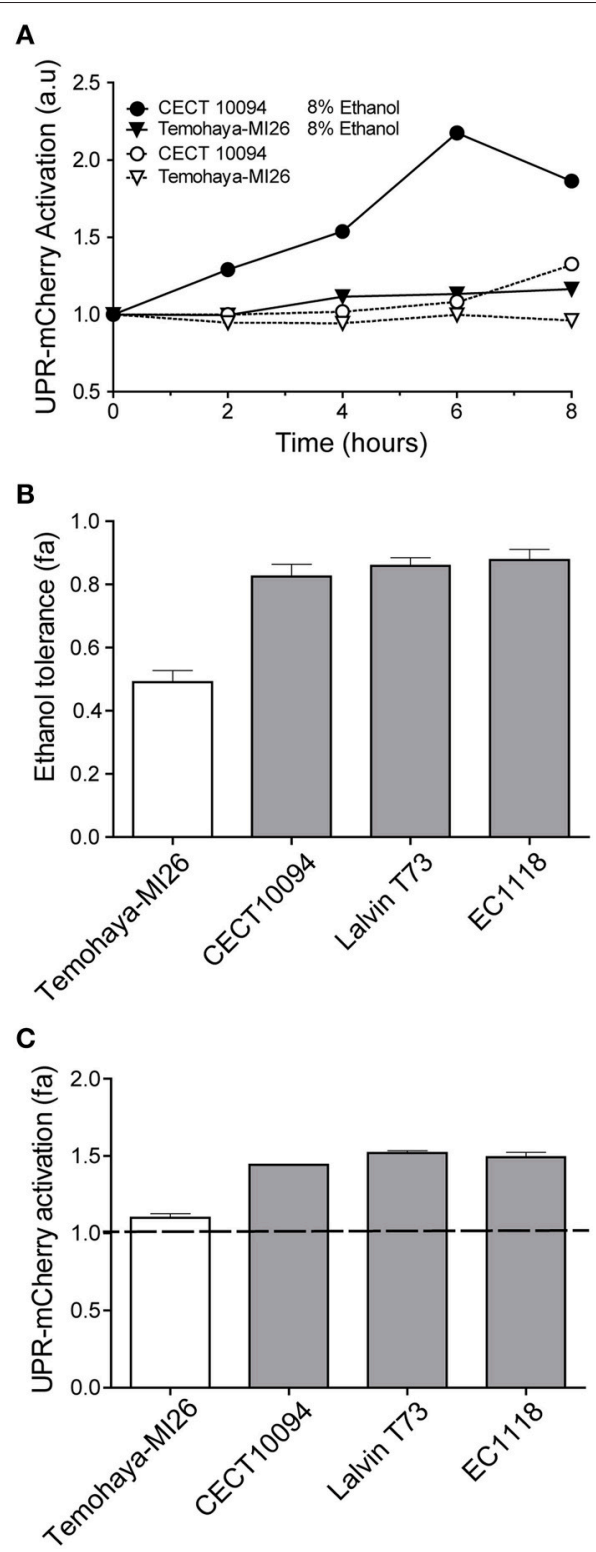

FIGURE 3 | Relation between ethanol tolerance and UPR signaling under ethanol stress. (A) UPR activation was analyzed measuring the fluorescence intensity by flow cytometry in Temohaya-MI26 and CECT10094 strains containing UPR mCherry reporter after resuspension of exponentially growing cells in control medium or containing 10\% of ethanol. Experiments were done in triplicate and data were normalized to its initial point. Calculated standard deviations did not exceed $5 \%$ of the average. (B) Ethanol tolerances in Temohaya-MI26, CECT 10094, Lalvin T73, and EC1118 strains were determined comparing yeast growth curve in GPY and GPY modified with $10 \%$ ethanol. Fractional area (fa) was calculated normalizing the area under the curve in GPY $10 \%$ ethanol respect to control. Three biological replicates were done and the mean value and standard deviation is shown. (C) UPR-mCherry activation in response to ethanol stress in Temohaya-MI26, CECT 10094, Lalvin T73, and EC1118 strains. The fractional area of mCherry intensity was obtained by the quotient between the area under the curve of the intensity of the reporter in GPY medium with ethanol (10\%) and control medium after $6 \mathrm{~h}$ of growth. The dotted line shows the threshold value above which the activation of the reporter is higher than in non-stress conditions (control). Gray bars represent the strains isolated from wine fermentation while the white bar shows the strain isolated from traditional fermentations. 
suggest that ethanol stress-essential genes may be more relevant for long-term adaptation or for subsequent stresses than the immediate response to stress, and that the network of induced genes is more important for adaptation to stress than the effect of a single gene. Besides we observed very dynamic responses (only 3.5 and $3.22 \%$ of commonality comparing both time points analyzed in the CECT10094 and Temohaya-MI26 strains, respectively) which hints that ethanol yeast tolerance is a procedure involving numerous events that interact over time.

According to the transcriptomic analysis, in the early ethanol stress stages, the ethanol-tolerant CECT10094 strain showed an overexpression of the genes related to transport and utilization of proline, synthesis of mono-unsaturated fatty acids, molecular chaperones, and response to oxidative stress, which were not seen in the less tolerant strain. Some of these genes have been previously described as key ethanol-tolerance genes that counteract the fluidizing effect of ethanol, inhibit aggregation during protein refolding and reduce the reactive oxidative species (ROS) produced during ethanol stress (Alexandre et al., 2001; Chandler et al., 2004). Several genes involved in NADH/NADPH regeneration were up-regulated in the CECT10094 strain in early stages of ethanol response, including ZWF1, SOL4, and ALD4. $\mathrm{NADPH}$ is required by glutathione and thioredoxin as a reducing agent to reduce oxidized glutathione (GSSG) and thioredoxin, these being key elements to counteract oxidative stress. In addition, NADPH is necessary to carry out NADH-dependent desaturation of stearic acid in oleic acid, and is considered the main determinant of ethanol tolerance in S. cerevisiae (You et al., 2003), formed by the catabolic membrane desaturase encoded by OLE1, which is also up-regulated in the CECT10094 strain. In contrast to other studies (Chandler et al., 2004; Ma and Liu, 2010a), our data and Shobayashi et al. (2007) have observed enhanced mRNA levels of OLE1 in the early stages of ethanol stress. This suggests that not only mRNA stability, translation, and enzyme reactions with carbon and oxygen sources regulate unsaturated fatty acid (UFA) biosynthesis (Martin et al., 2007), but transcription levels can also play a more important role than previously thought.

In the late ethanol stress response, we found that the genes related with energy generation (ATP1, ATP3, ATP4, ATP7, and $A T P 20)$ were significantly overexpressed in the most tolerant strain. This fact indicates that under ethanol stress conditions, increased ATP synthesis might be responsible for the activation of protein synthesis and proton transportation through the plasma membrane (Rosa and Sá-Correia, 1991). This is consistent with the overexpression of VMA genes (VMA10 and VMA8) that encode vacuolar $\mathrm{H}^{+}$-ATPase (V-ATPase), an electrogenic proton pump involved in vacuolar acidification and, therefore, in the compensation of $\mathrm{H}^{+}$entry induced by ethanol (Fujita et al., 2006). Higher ergosterol content in yeast has been associated with greater ethanol tolerance by preventing interdigitation and maintaining optimal membrane thickness (Vanegas et al., 2012). We noticed the overexpression of the genes related to the ergosterol pathway (ERG5, ERG11, and ERG20), which has not been previously observed in microarrays studies (Alexandre et al., 2001; Chandler et al., 2004; Teixeira et al., 2009; Ma and Liu, 2010a). It is noteworthy that the genes related with actin cytoskeleton organization, such as RDI1, RHO1, ARC19, and ARP2, were overexpressed in CECT10094. According to Kubota et al. (2004), the spatial organization of the F-actin cytoskeleton is transiently disrupted by the addition of ethanol. Thus, the CECT10094 strain can be expected to counteract the depolarization of F-actin more efficiently than the less tolerant strain to obtain a higher growth rate under stressful conditions.

Our results revealed that the retrotransposon-related Gene Ontology (GO) categories were highly up-regulated in CECT10094 strain, while the Temohaya-MI26 strain did not overexpress any retrotransposon-related gene. Little attention has been paid to retrotransposon-related gene activation after ethanol stress, probably due to the misinterpretation of transcriptomes and databases (Stanley et al., 2010b). Although the adaptive significance of this activation remains unclear, we observed overexpressed levels of GCN4, whose protein Gcn4p has been suggested to be a Tyl activator in stress (Morillon et al., 2002). Further work is required to determine whether an association exists between retrotransposons and the ethanol stress response in yeast.

\section{Ethanol Stress Triggers UPR Activation}

One very interesting finding among our results is the observation that the ethanol-tolerant CECT10094 strain overexpressed genes whose GO was related to protein catabolism and refolding in the late ethanol stress phase, mainly regulated by Haclp, a UPR-specific transcription factor that induces UPR target gene expression, including ER resident chaperones and critical protein-folding enzymes to restore ER protein-folding homeostasis (Travers et al., 2000; Ron and Walter, 2007; Kimata and Kohno, 2011). Indeed, we observed more genes with Haclp binding sites, in the most tolerant strain in both growth stages, as well as UPR target genes, such as LHS1, ERO1, and KAR2, which encoded redox proteins and ER chaperones and were up-regulated in the CECT10094 strain. The correlation between ethanol and unfolded protein tolerance (Table 4) suggests that strains with an enhanced unfolded protein response also increase ethanol resistance. Furthermore, our results confirmed that ethanol triggers the UPR response in the cell and that the conjunction between UPR response and ethanol tolerance may be an interesting way to explain how yeast overcomes this stress. To date, ethanol adaptation has been related with the coordinate action of transcription factors Msn2/4p, Yap1p, and Hsf1p to control general stress, oxidative stress and heat shock responses, respectively, in yeast (Ma and Liu, 2010a,b).

Ethanol and UPR signaling activation has been previously correlated in human cells (Pandol et al., 2010; Ji, 2012). The unfolded protein response (UPR) is a complex pathway triggered by ER stress to enhance and restore the protein-folding and secretory capacity of the ER, hence its activation under ethanol stress is conserved across vertebrates. Likewise, alcohol damages mammalian cells and induces numerous pathological stress responses, such as the ER stress response, which has recently emerged as a novel mechanism for pancreas and liver disease in chronic alcoholism. In fact, a recent study has correlated pancreas disease with an insufficient UPR response, and ER stress-induced overproduction of lipids can lead to fatty liver in 
alcoholic patients (Ji and Kaplowitz, 2003; Pandol et al., 2010). These data support our findings and suggest that UPR activation after UPR stress can be conserved in eukaryotes.

Interestingly, not all strains activate UPR at the same level after ethanol stress. This suggests that the strains that have been adapting their physiology to elevated amounts of ethanol, as it is found in fermentative process as winemaking, rely on UPR signaling to resist this suboptimal condition. All this data pointed out that there are possibilities to improve the ethanol tolerance of selected yeast starters via the enhancement of UPR. Also, the important differences that can be observed among the different strains suggest that UPR pathway can be a key modulator to adapt yeast cells to the different environments.

In summary, this work shed light on the ethanol transcriptional stress response, demonstrates the role of the UPR pathway under ethanol stress and links this response to an enhanced ethanol tolerance. These data uncover potential applications to increase ethanol tolerance of yeasts. Many industrial applications, such as winemaking, depend on yeast tolerance to ethanol. Thus our research opens new line of possibilities related to UPR pathway enhancement to increase ethanol stress resistance. Either selecting natural or engineered strains with elevated UPR response will be a new field for industrial yeast strain improvement. Future experiments will shed more light on the specific signal that triggers UPR activation during ethanol stress.

\section{AUTHOR CONTRIBUTIONS}

$\mathrm{AQ}, \mathrm{EN}$, and RP conceived and designed the experiments. $\mathrm{EN}$ and RK performed the experiments. AQ, EN, RP, and RK analyzed the data. EN contributed reagents/materials/analysis tools. AQ, EN, and RP wrote the paper.

\section{ACKNOWLEDGMENTS}

The authors thank Sergi Puig and Eladio Barrio for their valuable comments on the manuscript (Instituto de Agroquímica y Tecnología de los Alimentos, IATA-CSIC, Paterna, Spain) and SCSIE of the University of Valencia for support with the cytometry flow analysis. EN wishes to Jordi Tronchoni for his technical help in the microarrays experiments and Vicente Andreu for his valuable support. EN was supported

\section{REFERENCES}

Albano, E. (2006). Alcohol, oxidative stress and free radical damage. Proc. Nutr. Soc. 65, 278-290. doi: 10.1079/PNS2006496

Alexandre, H., Ansanay-Galeote, V., Dequin, S., and Blondin, B. (2001). Global gene expression during short-term ethanol stress in Saccharomyces cerevisiae. FEBS Lett. 498, 98-103. doi: 10.1016/S0014-5793(01)02503-0

Alexandre, H., Rousseaux, I., and Charpentier, C. (1994a). Ethanol adaptation mechanisms in Saccharomyces cerevisiae. Biotechnol. Appl. Biochem. 20(Pt 2), 173-183.

Alexandre, H., Rousseaux, I., and Charpentier, C. (1994b). Relationship between ethanol tolerance, lipid composition and plasma membrane fluidity in by a FPU grant from the Ministerio de Educación, Cultura y Deporte (ref. AP2009-0787). RP was supported by a JAEDOC postdoctoral program (IATA-CSIC), co-funded by FSE. This work has been supported by grants AGL2012-39937-C0201 from the Spanish Government, FEDER and Generalitat Valenciana PROMETEOII/2014/042 to AQ. We acknowledge support of the publication fee by the CSIC Open Access Publication Support Initiative through its Unit of Information Resources for Research (URICI).

\section{SUPPLEMENTARY MATERIAL}

The Supplementary Material for this article can be found online at: http://journal.frontiersin.org/article/10.3389/fmicb. 2016.00189

Supplementary Figure 1 | Microarray data validation by qPCR (eps file,.eps). mRNA of significantly activated genes ACS1, OPI3, TFS1 (in Temohaya-MI26 and CECT10094) and ERO1, ALD4 y HSC82 (in CECT10094) was measured by RT-qPCR, using $A C T 1$ and $18 \mathrm{~S}$ ribosomal gene as reference. High relation between microarray and RT-qPCR data was observed, showing a Spearman correlation coefficient ( $\rho$ ) of $0.833(p=0.008 ;$ A). Also, Bland-Altman analysis to study method concordance showed a high similar outcome of both techniques (95\% confidence interval; B). The $x$-axis represents the average fold change of each sample measured by quantitative reverse transcription PCR (RT-qPCR) and microarrays. The $y$-axis is the difference in fold change calculated by microarray measurements minus the RT-qPCR measurements for each sample. The solid line $(y=-0.52)$ is the mean difference in fold change of all the samples. The two dotted lines represent 1.96 standard deviations from the mean difference.

Supplementary Table 1 | Gene expression under ethanol stress (excel file,.xlsx). Comparison of the mRNA expression of S. cerevisiae CECT10094 and Temohaya-MI26 by fold changes after $1 \mathrm{~h}$ and after exponential phase after addition of ethanol to the GPY medium. The expressions for each gene at each time point were represented in the relative fold changes against themselves under non-stressed conditions. Differentially expressed genes were identified as $\log _{2}$ (ratio) values of the more than 1.96 standard deviations from the mean (at the 95\% confidence level). Red indicates overexpression, green is repressed expression, and yellow implies no significant changes.

Supplementary Table 2 | Up-regulated genes in S. cerevisiae CECT 10094 and Temohaya-MI26 after $1 \mathrm{~h}$ and after exponential phase of ethanol stress (excel file,.xlsx). Promoter regions were analyzed using Regulatory Sequence Analysis Tools (RSAT) (http://rsat.ulb.ac.be/rsat). The number of binding sites for transcription factors involved in various stress responses, such as Msn2p/Msn4p for general stress, Hac1p for unfolded protein response, Hsf1p for heat stress, and Yap1p for oxidative stress, are shown. The number of STRE elements in their promoter regions, implicated in a general stress response in yeast, was also analyzed. Those genes previously found to be up-regulated under ethanol stress are underlined.
Saccharomyces cerevisiae and Kloeckera apiculata. FEMS Microbiol. Lett. 124, 17-22. doi: 10.1111/j.1574-6968.1994.tb07255.x

Alper, H., Moxley, J., Nevoigt, E., and Fink, G. R. (2006). Stephanopoulos, G. Engineering yeast transcription machinery for improved ethanol tolerance and production. Science 314, 1565-1568. doi: 10.1126/science.11 31969

Ansanay-Galeote, V., Blondin, B., Dequin, S., and Sablayrolles, J. M. (2001). Stress effect of ethanol on fermentation kinetics by stationary-phase cells of Saccharomyces cerevisiae. Biotechnol. Lett. 23, 677-681. doi: 10.1023/A:1010396232420

Arroyo-López, F. N., Salvadó, Z., Tronchoni, J., Guillamón, J. M., Barrio, E., and Querol, A. (2010). Susceptibility and resistance to ethanol in Saccharomyces 
strains isolated from wild and fermentative environments. Yeast 27, 1005-1015. doi: 10.1002/yea.1809

Belloch, C., Orlic, S., Barrio, E., and Querol, A. (2008). Fermentative stress adaptation of hybrids within the Saccharomyces sensu stricto complex. Int. J. Food. Microbiol. 122, 188-195. doi: 10.1016/j.ijfoodmicro.2007. 11.083

Brooks, P. J. (2000). Brain atrophy and neuronal loss in alcoholism, a role for DNA damage? Neurochem. Int. 37, 403-412. doi: 10.1016/S0197-0186(00)00051-6

Causton, H. C., Quackenbush, J., and Brazma, A. (2003). "Image processing, normalisation and data transformation," in Microarray Gene Expression Data Analysis, A Beginner's Guide, ed M. A. Malden (Oxford, UK: Blackwell Publishing), 51-70.

Chandler, M., Stanley, G. A., Rogers, P., and Chambers, P. (2004). A genomic approach to defining the ethanol stress response in the yeast Saccharomyces cerevisiae. Ann. Microbiol. 54, 427-454.

Combina, M., Pérez-Torrado, R., Tronchoni, J., Belloch, C., and Querol, A. (2012). Genome-wide gene expression of a natural hybrid between Saccharomyces cerevisiae and S. kudriavzevii under enological conditions. Int. J. Food Microbiol. 157, 340-345. doi: 10.1016/j.ijfoodmicro.2012.06.001

Cross, F. R. (1997). 'Marker swap' plasmids, convenient tools for budding yeast molecular genetics. Yeast 13, 647-653.

Forgac, M. (1998). Structure, function and regulation of the vacuolar (H+)ATPases. FEBS Lett. 440, 258-263. doi: 10.1016/S0014-5793(98)01425-2

Fujita, K., Matsuyama, A., Kobayashi, Y., and Iwahashi, H. (2006). The genomewide screening of yeast deletion mutants to identify the genes required for tolerance to ethanol and other alcohols. FEMS Yeast Res. 6, 744-750. doi: 10.1111/j.1567-1364.2006.00040.x

Gasch, A. P., Spellman, P. T., Kao, C. M., Carmel-Harel, O., Eisen, M. B., Storz, G., et al. (2000). Genomic expression programs in the response of yeast cells to environmental changes. Mol. Biol. Cell. 11, 4241-4257. doi: 10.1091/mbc.11.12. 4241

Hirasawa, T., Yoshikawa, K., Nakakura, Y., Nagahisa, K., Furusawa, C., Katakura, Y., et al. (2007). Identification of target genes conferring ethanol stress tolerance to Saccharomyces cerevisiae based on DNA microarray data analysis. J. Biotechnol. 131, 34-44. doi: 10.1016/j.jbiotec.2007.05.010

Hoek, J. B., and Pastorino, J. G. (2002). Ethanol, oxidative stress, and cytokineinduced liver cell injury. Alcohol 27, 63-68. doi: 10.1016/S0741-8329(02)00215$\mathrm{X}$

Ji, C., and Kaplowitz, N. (2003). Betaine decreases hyperhomocysteinemia, endoplasmic reticulum stress, and liver injury in alcohol-fed mice. Gastroenterology 124, 1488-1499. doi: 10.1016/S0016-5085(03)00276-2

Ji, C. (2012). Mechanisms of alcohol-induced endoplasmic reticulum stress and organ injuries. Biochem. Res. Int. 2012, 12. doi: 10.1155/2012/216450

Kimata, Y., and Kohno, K. (2011). Endoplasmic reticulum stress-sensing mechanisms in yeast and mammalian cells. Curr. Opin. Cell Biol. 23, 135-142. doi: 10.1016/j.ceb.2010.10.008

Kubota, S., Takeo, I., Kume, K., Kanai, M., Shitamukai, A., Mizunuma, M., et al. (2004). Effect of ethanol on cell growth of budding yeast, genes that are important for cell growth in the presence of ethanol. Biosci. Biotechnol. Biochem. 68, 968-972. doi: 10.1271/bbb.68.968

Lamarche, F., Gonthier, B., Signorini, N., Eysseric, H., and Barret, L. (2003). Acute exposure of cultured neurones to ethanol results in reversible DNA singlestrand breaks; whereas chronic exposure causes loss of cell viability. Alcohol Alcohol. 38, 550-558. doi: 10.1093/alcalc/agg118

Lambert, R. J., and Pearson, J. (2000). Susceptibility testing, accurate and reproducible minimum inhibitory concentration (MIC) and noninhibitory concentration (NIC) values. J. Appl. Microbiol. 88, 784-790. doi: 10.1046/j.1365-2672.2000.01017.x

Li, B. Z., Cheng, J. S., Ding, M. Z., and Yuan, Y. J. (2010). Transcriptome analysis of differential responses of diploid and haploid yeast to ethanol stress. J. Biotechnol. 148, 194-203. doi: 10.1016/j.jbiotec.2010.06.013

Llopis, S., Querol, A., Heyken, A., Hube, B., Jespersen, L., Fernández-Espinar, M. T., et al. (2012). Transcriptomics in human blood incubation reveals the importance of oxidative stress response in Saccharomyces cerevisiae clinical strains. BMC Genomics 13:419. doi: 10.1186/1471-2164-13-419

Ma, M., and Liu, L. Z. (2010b). Quantitative transcription dynamic analysis reveals candidate genes and key regulators for ethanol tolerance in Saccharomyces cerevisiae. BMC Microbiol. 10:169. doi: 10.1186/1471-2180-10-169
Ma, M., and Liu, Z. L. (2010a). Mechanisms of ethanol tolerance in Saccharomyces cerevisiae. Appl. Microbiol. Biotechnol. 87, 829-845. doi: 10.1007/s00253-0102594-3

Marks, V. D., Ho Sui, S. J., Erasmus, D., van der Merwe, G. K., Brumm, J., Wasserman, W. W., et al. (2008). Dynamics of the yeast transcriptome during wine fermentation reveals a novel fermentation stress response. FEMS Yeast Res. 8, 35-52. doi: 10.1111/j.1567-1364.2007.00338.x

Martin, C. E., Oh, C. S., and Jiang, Y. (2007). Regulation of long chain unsaturated fatty acid synthesis in yeast. Biochim. Biophys. 1771, 271-285. doi: 10.1016/j.bbalip.2006.06.010

Merksamer, P. I., Trusina, A., and Papa, F. R. (2008). Real-time redox measurements during endoplasmic reticulum stress reveal interlinked protein folding functions. Cell 135, 933-947. doi: 10.1016/j.cell.2008.10.011

Morillon, A., Bénard, L., Springer, M., and Lesage, P. (2002). Differential effects of chromatin and Gcn4 on the 50-fold range of expression among individual yeast Ty1 retrotransposons. Mol. Cell. Biol. 22, 2078-2088. doi: 10.1128/MCB.22.7.2078-2088.2002

Oliveira, B. M., Barrio, E., Querol, A., and Pérez-Torrado, R. (2014). Enhanced enzymatic activity of glycerol-3-phosphate dehydrogenase from the cryophilic Saccharomyces kudriavzevii. PLoS ONE 9:e87290. doi: 10.1371/journal.pone.0087290

Pandol, S. J., Gorelick, F. S., Gerloff, A., and Lugea, A. (2010). Alcohol abuse, endoplasmic reticulum stress and pancreatitis. Dig. Dis. 28, 776-782. doi: $10.1159 / 000327212$

Ron, D., and Walter, P. (2007). Signal integration in the endoplasmic reticulum unfolded protein response. Nat. Rev. Mol. Cell Biol. 8, 519-529. doi: $10.1038 / \mathrm{nrm} 2199$

Rosa, M. F., and Sá-Correia, I. (1991). In vivo activation by ethanol of plasma membrane ATPase of Saccharomyces cerevisiae. Appl. Environ. Microbiol. 57, 830-835.

Rosa, M. F., and Sa-Correia, I. (1996). Intracellular acidification does not account for inhibition of Saccharomyces cerevisiae growth in the presence of ethanol. FEMS Microbiol. Lett. 135, 271-274. doi: 10.1111/j.1574-6968.1996.tb0 8000.x

Rossignol, T., Dulau, L., Julien, A., and Blondin, B. (2003). Genome-wide monitoring of wine yeast gene expression during alcoholic fermentation. Yeast 20, 1369-1385. doi: 10.1002/yea.1046

Santa María, J. (1968). Sacch, hispanica, nov. spec., nueva especie de levadura de "flor." Bol. Inst. Nac. Inv. Agronom. (Madrid) 58, 21-32.

Shobayashi, M., Ukena, E., Fujii, T., and Iefuji, H. (2007). Genome-wide expression profile of sake brewing yeast under shaking and static conditions. Biosci. Biotechnol. Biochem. 71, 323-335. doi: 10.1271/bbb.60190

Stanley, D., Chambers, P. J., Stanley, G. A., Borneman, A., and Fraser, S. (2010a). Transcriptional changes associated with ethanol tolerance in Saccharomyces cerevisiae. Appl. Microbiol. Biotechnol. 88, 231-239. doi: 10.1007/s00253-0102760-7

Stanley, D., Fraser, S., Stanley, G. A., and Chambers, P. J. (2010b). Retrotransposon expression in ethanol-stressed Saccharomyces cerevisiae. Appl. Microbiol. Biotechnol. 87, 1447-1454. doi: 10.1007/s00253-0102562-y

Supek, F., Bošnjak, M., Škunca, N., and Šmuc, T. (2011). REVIGO summarizes and visualizes long lists of gene ontology terms. PLOS ONE 6:e21800. doi: 10.1371/journal.pone.0021800

Takahashi, T., Shimoi, H., and Ito, K. (2001). Identification of genes required for growth under ethanol stress using transposon mutagenesis in Saccharomyces cerevisiae. Mol. Genet. Genomics 265, 1112-1119. doi: 10.1007/s0043 80100510

Takemura, R., Inoue, Y., and Izawa, S. (2004). Stress response in yeast mRNA export factor, reversible changes in Rat8p localization are caused by ethanol stress but not heat shock. J. Cell Sci. 117, 4189-4197. doi: 10.1242/jcs.01296

Teixeira, M. C., Raposo, L. R., Mira, N. P., Lourenco, A. B., and Sá-Correia, I. (2009). Genome-wide identification of Saccharomyces cerevisiae genes required for maximal tolerance to ethanol. Appl. Environ. Microbiol. 75, 5761-5772. doi: 10.1128/AEM.00845-09

Travers, K. J., Patil, C. K., Wodicka, L., Lockhart, D. J., Weissman, J. S., and Walter, P. (2000). Functional and genomic analyses reveal an essential coordination between the unfolded protein response and ER-associated degradation. Cell 101, 249-258. doi: 10.1016/S0092-8674(00)80835-1 
Vanegas, J. M., Contreras, M. F., Faller, R., and Longo, M. L. (2012). Role of unsaturated lipid and ergosterol in ethanol tolerance of model yeast biomembranes. Biophys. J. 102, 507-516. doi: 10.1016/j.bpj.2011. 12.038

Yoshikawa, K., Tanaka, T., Furusawa, C., Nagahisa, K., Hirasawa T., and Shimizu, H. (2009). Comprehensive phenotypic analysis for identification of genes affecting growth under ethanol stress in Saccharomyces cerevisiae. FEMS Yeast Res. 9, 32-44. doi: 10.1111/j.1567-1364.2008.00456.x

You, K. M., Rosenfield, C. L., and Knipple, D. C. (2003). Ethanol tolerance in the yeast Saccharomyces cerevisiae is dependent on cellular oleic acid content. Appl. Environ. Microbiol. 69, 1499-1503. doi: 10.1128/AEM.69.3.1499-1503.2003
Conflict of Interest Statement: The authors declare that the research was conducted in the absence of any commercial or financial relationships that could be construed as a potential conflict of interest.

Copyright (c) 2016 Navarro-Tapia, Nana, Querol and Pérez-Torrado. This is an open-access article distributed under the terms of the Creative Commons Attribution License (CC BY). The use, distribution or reproduction in other forums is permitted, provided the original author(s) or licensor are credited and that the original publication in this journal is cited, in accordance with accepted academic practice. No use, distribution or reproduction is permitted which does not comply with these terms. 